\title{
Epigenetic Modifications upon Senescence of Mesenchymal Stem Cells
}

\author{
Julia Franzen ${ }^{1,2}$ - Wolfgang Wagner ${ }^{1,2} \cdot$ Eduardo Fernandez-Rebollo ${ }^{1,2}$
}

Published online: 1 June 2016

(C) Springer International Publishing AG 2016

\begin{abstract}
Cellular senescence is a continuous and highly organized process that alters the intricate genomic network in order to maintain cellular homeostasis. It occurs in all primary cell cultures - including mesenchymal stem cells (MSCs), which are concurrently tested for a wide variety of clinical applications. Differentiation potential as well as paracrine secretion of MSCs is severely affected by cellular senescence. There is a growing perception that nuclear reorganization and epigenetic modifications contribute to trigger and maintain functional differences in long-term culture. In this review, we discuss molecular and epigenetic aspects that evoke functional changes in cellular aging-indicating that the underlying process is not only an accumulation of cellular defects, but rather epigenetically orchestrated.
\end{abstract}

Keywords Cellular aging $\cdot$ Epigenetics $\cdot$ Mesenchymal stem cell and senescence

\section{Introduction}

Mesenchymal stem cells (MSCs) can be differentiated towards osteocytes, adipocytes, and chondrocytes. They can be easily isolated from various tissues and harbor both

This article is part of the Topical Collection on Age-related Stem Cell Modifiers

Eduardo Fernandez-Rebollo

efernandez@ukaachen.de

1 Helmholtz-Institute for Biomedical Engineering, Stem Cell Biology and Cellular Engineering, RWTH Aachen University Medical School, Pauwelsstraße 20, 52074 Aachen, Germany

2 Institute for Biomedical Engineering-Cell Biology, RWTH Aachen Medical School, 52074 Aachen, Germany regenerative and immunomodulatory potential. MSCs have been tested in a multitude of clinical trials - the clinical relevance is often not unequivocally demonstrated, but at least, there were so far no severe side effects [1]. Ex vivo expansion of MSCs is indispensable for obtaining sufficient amounts of cells for cellular therapies or tissue engineering. Nevertheless, MSCs have a limited lifespan in vitro and enter replicative senescence after a predefined number of cell divisions. Replicative senescence of MSCs is not only reflected in decay of proliferation but also in continuous changes of cellular morphology, size, and loss of differentiation potential [2]. Thus, senescence is of major significance for the therapeutic potential of MSCs and needs to be considered in terms of quality control.

Replicative senescence was formally described half a century ago by Leonard Hayflick in human diploid fibroblasts as a permanent cell cycle arrest after a limited number of cell divisions in culture [3]. It is generally assumed that this type of cellular aging is caused by telomere attrition, a natural process occurring with each cell cycle [4]. Alternatively, cellular senescence can be induced by DNA damage through external stress like reactive oxygen species or the activation of oncogenes [5, 6]. Cellular senescence is often considered to be a hallmark of aging, suggesting that the two processes might be related [7].

At the molecular level, cellular senescence seems to be particularly mediated by two tumor suppressor pathways: p53/p21CIP1 and p16INK4A/Rb [8]. Both pathways are activated upon the entry into senescence. The $\mathrm{p} 53$ protein proceeds in activating transcriptional targets, such as $\mathrm{p} 21$, which finally induce cell cycle arrest [9]. The p16 cyclin-dependent kinase inhibitor represses the phosphorylation of the retinoblastoma protein $(\mathrm{Rb})$, thereby generating its active, hypophosphorylated form. In its activated form $\mathrm{Rb}$ binds to the E2F protein family members to inhibit their transcriptional 
targets [10]. These targets constitute the majority of effectors required for cell-cycle progression. Senescent cells encompass a phenotype characterized by specific features in gene expression, senescence-associated beta galactosidase activity, DNA methylation, histone modifications, senescence-associated heterochromatic foci, DNA damage foci, and a senescenceassociated secretory phenotype $[11 \bullet \bullet]$. Modulation of these pathways remains to be elucidated. However, several epigenetic mechanisms have been identified to play a major role in regulation of senescence (Fig. 1). In this review, we want to recapitulate the current state of research on epigenetic modifications in human MSCs upon senescence.

\section{Nuclear Reorganization: Chromatin Remodeling and the Nuclear Lamina}

The three-dimensional nuclear organization of the chromatin is fundamental for epigenetic control of gene expression and genome stability [12]. In proliferating cells, heterochromatin domains of different chromosomes are attached to the nuclear lamina - the complex protein scaffold that lies under the inner nuclear membrane [13]. Anchoring of heterochromatin to the nuclear lamina facilitates and keeps interaction of specific chromatin domains. However, during senescence, the attachment of specific heterochromatic regions to the lamina is lost, and nuclear architecture is reshaped [14]. Repositioning of the heterochromatin regions from the periphery to the nuclear center generates novel genetic interaction domains, largely known as senescence-associated heterochromatic foci (SAHF), which are usually associated with repressive histone marks, such as histone 3 lysine 9 trimethylation (H3K9me3) [10]. A whole-genome chromosome conformation capture (Hi-C) analysis in human senescent fibroblasts has unraveled that certain topologically associated domains (TAD) are relocated acquiring novel short-range interactions while losing long-range interactions within chromosomes [15]. Another Hi-C study of senescent fibroblasts has shown that the local genetic interactions within SAHF domains are weakened [14]. These findings raise the question if a similar rearrangement of TADs, as well as loss of interactions within the SAHF, occurs also in other cell types upon senescence.

Mesenchymal stem cells encompass the global threedimensional reorganization of the nuclear framework during senescence. They suffer a broad spatial disorganization of the nuclear lamina upon cellular aging [16]. Concretely, lamin A and $\mathrm{B}$, the main components of the nuclear lamina, are deregulated during cellular aging. Lamin A is redistributed forming accumulations that force the nuclear lamina to bend [17]. While lamin B and its associated receptor, which anchors heterochromatin to the lamina, are downregulated during senescence [18•]. In addition, lamina-associated domains (LADs) become hypomethylated while regions outside LADs are mainly hypermethylated $[18 \bullet, 19]$. Rebuilding of the nuclear scaffold during senescence is mirrored in the Werner syndrome - a premature aging disorder caused by deficiency of Werner syndrome RecQ helicase-like (WRN) protein [20•]. MSCs lacking WRN show a global loss of

Fig. 1 The epigenetic hallmarks of senescence in MSCs. Schematic representation of the different epigenetic mechanisms governing senescence in mesenchymal stem cells

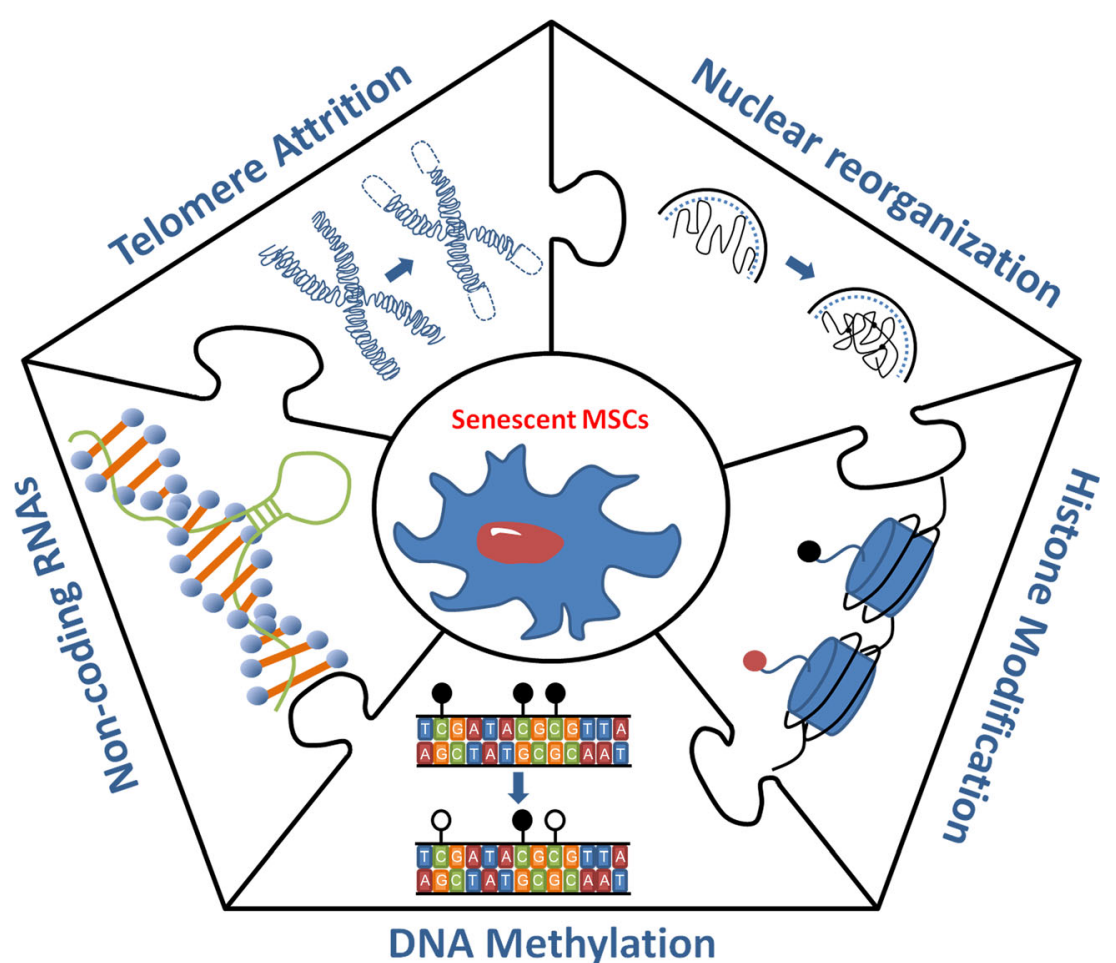


H3K9me3 and a deregulation of heterochromatin proteins, such as suppressor of variegation 3-9 homolog 1 (SUV39HI) and heterochromatin protein $1 \alpha(H P 1 \alpha)$, or nuclear laminaassociated polypeptide $2 \beta(L A P 2 \beta)$, leading to a global nuclear disorganization.

The fine regulation of the chromatin reorganization cascade is reflected by the modulation of Brahma-related gene 1 (BRG1), the ATPase subunit of the chromatin remodeling complex switch/sucrose non-fermentable (SWI/SNF). BRG1 deregulation induces senescence in MSCs through suppression of NANOG transcription - a keystone of the transcriptional circuitry governing stemness [21, 22]. BRG1 downregulation increases the recruitment of Rb and DNA methyltransferase 1 (DNMT1) at the NANOG promoter, thereby inducing methylation and silencing NANOG at the transcriptional level. Moreover, BRG1 overexpression increases BRG1 occupancy at the NANOG promoter, inducing chromatin compaction and recruiting histone deacetylases (HDAs) [23]. Taken together, senescence of MSCs has enormous impact on nuclear organization - and disorganizing the proper nuclear structure may per se entail genomic instability.

\section{Telomere Attrition-a Hallmark for Replicative Senescence}

Telomeres and telomere-associated proteins form a unique structure that protects the chromosome ends from being recognized as DNA double-strand breaks $[24,25]$. This is relevant for escape of DNA damage response (DDR) and protection from aberrant fusion and recombination events, thereby preserving genome integrity [26]. Maintenance of telomere length is regulated by the telomerase ribonucleoprotein complex consisting of telomerase reverse transcriptase ( $h T E R T$ ) and a long non-coding RNA (lncRNA) which is named telomeric repeat-containing RNA $(T E R R A)[11 \bullet \cdot]$. Interestingly, this lncRNA is deregulated during senescence in various cell types [27]. Long-term expansion of MSCs is reflected by telomere attrition, since they have insufficient telomerase activity to overcome the progressive telomere erosion [28]. Supporting these findings, overexpression of hTERT in senescent MSCs partially counteracts replicative senescence $[29,30]$. On the other hand, long-term cultures of human stem cells, including MSCs, have shown several types of chromosomal abnormalities that could compromise genome integrity and their clinical applicability [31, 32]. MSCs reveal a high percentage of aneuploid cells upon replicative senescence or oxidative stress. Notably, hTERT overexpression seems to also reduce the levels of aneuploidy and partially prevents the entrance to senescence [33•].

Consequently, telomeres attrition has been considered an emblem of cellular aging since the erosion of the protective caps of the telomeres ignites replicative senescence.

\section{DNA Methylation Changes During Cellular Senescence}

In somatic cells, DNA methylation (DNAm) occurs almost exclusively in the context of cytosine-guanine dinucleotides - so called $\mathrm{CpG}$ sites. The methylation pattern of $\mathrm{CpG}$ sites is maintained and modified by DNA methyltransferases (DNMTs). There is no direct correlation between DNA methylation and gene expression levels, albeit methylation of promoter regions being mostly associated with gene silencing [34]. Overall, the global DNAm patterns of MSCs are maintained during long-term culture. In particular, specific $\mathrm{CpG}$ sites with stable methylation during in vitro culture can resemble the cell type and tissue of origin $[35,36]$. On the other hand, several CpG sites show DNAm changes upon culture expansion $[37 \bullet, 38]$. Some of these changes are highly reproducible and show linear correlations to cumulative population doublings, passage numbers, or even time of in vitro culture. Therefore, senescence-associated DNAm changes of six specific $\mathrm{CpG}$ sites can be used to estimate the state of senescence of MSCs [39, 40].

In general, DNAm changes of MSCs in replicative senescence seem to be related to age-associated DNAm changes $[19,38]$, indicating that both aging processes might be modulated by similar epigenetic mechanisms. However, ageassociated and senescence-associated DNA methylation changes are not identical since specific senescenceassociated DNAm changes are not affected by donor age [40] and specific age-associated DNAm changes are hardly affected by in vitro culture [41-43]. Noteworthy, induced pluripotent stem cells (iPSCs) do not undergo replicative senescence, and reprogramming into iPSCs is associated with telomere extension. Furthermore, virtually all senescenceassociated as well as age-associated DNAm changes seem to be reversed in the process of reprogramming into iPSCs [37•]. Only after re-differentiation of iPSCs towards iPSC derived MSCs, the cells gradually reacquire the senescence-associated DNAm changes [44•].

Taken together, MSCs show DNAm as a mutual epigenetic regulator during in vitro and in vivo aging. The fact that senescence-associated DNAm modifications are acquired in a highly reproducible manner and that they can be reversed by reprogramming into iPSCs indicates that senescence of MSCs is somehow tightly regulated.

Differentiation of MSCs towards osteogenic and adipogenic lineages is impaired during in vitro expansion $[45,46]$. It is conceivable, that senescence-associated DNAm patterns impact on the expression of relevant genes, which are important for differentiation [38, 47]. Several transcription factors involved in differentiation, stem cell development, and tumorigenesis have significant enrichment of binding motives in differentially methylated regions as well as in promoters of differentially expressed genes during 
senescence [18•]. However, the promoter methylation of relevant genes for osteogenesis, such as runt-related transcription factor $2(R U N X 2)$ and alkaline phosphatase $(A L P)$ [48] or adipogenesis, such as fatty acid binding protein 4 (FABP4) and lipoprotein lipase $(L P L)$ [46], remains unchanged in the course of cellular senescence. Therefore, DNAm is a relevant epigenetic mechanism in the differentiation commitment, but not essential. Indeed, altered DNAm patterns might influence other epigenetic mechanisms, such as histone modifications or non-coding RNAs, playing an indirect role on the impaired MSC differentiation potential in senescence. The functional relevance of senescence-associated DNAm changes is therefore not unequivocally demonstrated - but they can clearly be utilized as reliable biomarker for replicative senescence.

\section{Histone Posttranslational Modifications Govern Functional Changes in Senescence}

An additional layer of epigenetic regulation is mediated by histone posttranslational modifications (PTMs), such as methylation and acetylation of histone tails. These modifications are tightly controlled, and govern chromatin accessibility and thereby gene expression [49]. Different combinations of PTMs on histones determine both how and when the packaged DNA can be accessed [50,51].

Histone acetylation is catalyzed by histone acetyltransferases (HATs) and histone deacetylases (HDACs), which add or remove acetyl groups, respectively. The activity of HDACs and HATs is dynamic and tightly regulated since these transient marks regulate cell-specific gene expression. Treatment with HDAC inhibitors indicates that HDAC activity is essential for maintenance of self-renewal, multipotency, and differentiation potential of MSCs [52]. Expression changes of several genes that are relevant for self-renewal (OCT4 and SOX2) or for maintenance of telomeres ( $h T E R T$ ) seem to be associated with a decreased acetylation of histone 3 at lysine 9 or 14 (H3K9ac or $\mathrm{H} 3 \mathrm{~K} 14 \mathrm{ac}$ ) in the promoter regions [48]. H3K9ac and $\mathrm{H} 3 \mathrm{~K} 14 \mathrm{ac}$ are required for the recruitment of different transcription factors [53] and the chromatin remodeling complex SWI/SNF, to initiate the transcription [54]. HDACs are generally downregulated upon senescence of MSCs, and this entails decreased expression of the polycomb group genes (PcGs) and an increased expression of Jumonji domain containing three (JMJD3) proteins, both involved in cell cycle control [55]. Thus, histone acetylation plays an important role for balancing genes involved in stemness as well as cell cycle progression.

In contrast to histone acetylation, histone methylation can serve as a context-dependent repressive or permissive mark, catalyzed by histone methyltransferases and histone demethylases. Histone methylation generally regulates gene expression indirectly by altering interactions with other proteins. Long-term culture of MSCs leads to an enrichment in repressive histone PTMs, such as histone 3 lysine 4 and lysine 27 trimethylation (H3K4me3 and H3K27me3, respectively) [56, 57]. Interestingly, replicative senescenceassociated hypermethylated loci of MSCs show significant enrichment not only in repressive histone marks H3K4me3 and $\mathrm{H} 3 \mathrm{~K} 27 \mathrm{me} 3$ but also in the active histone mark H3K4me1 [18•, 57]. In contrast, during cellular senescence, the active chromatin mark $\mathrm{H} 3 \mathrm{~K} 4 \mathrm{me} 1$ is enriched in hypomethylated sites [58•].

Thus, upon senescence of MSCs repressing histone, PTMs correlate with repressing DNA methylation changes, hence reinforcing and complementing each other. On the other hand, the active chromatin mark $\mathrm{H} 3 \mathrm{~K} 4 \mathrm{me} 1$ is associated with hypoor hypermethylated loci during in vivo (cellular) or in vitro (replicative) senescence, accordingly.

\section{Non-Coding RNAs Affect Senescence-from Short to Long Transcripts}

MicroRNAs (miRNAs) are short, non-protein-coding RNA species (ncRNAs) that have a pivotal role in posttranscriptional regulation of gene expression. miRNAs bind to the $3^{\prime}$ untranslated region (UTR) of their target transcripts, resulting in reduction of gene expression by mRNA degradation or translational blocking [59]. A single miRNA is able to regulate hundreds of different mRNA targets in various cellular functions, including metabolism, proliferation, apoptosis, senescence, and differentiation [60]. More than 45 miRNAs have been associated to replicative senescence of human MSCs [61-63]. The let-7 family of miRNAs and miRNAsbinding protein Argonaute 2 (AGO2) are critical for senescence induction by facilitating transcriptional gene silencing of E2F-regulated proliferation promoting genes [61, 64]. On the other hand, the miR-10a upregulation results in increased differentiation to adipogenic, osteogenic, and chondrogenic lineages and in reduced cellular aging of MSCs through the repression of the cell cycle regulator Kruppel-like factor 4 (KLF4) [65]. Evidencing the crosstalk between different epigenetic mechanisms, the miR-199b-5p suppresses laminin subunit Gamma 1 (LAMC1) expression, main component of the nuclear organization $[66 \bullet, 67]$. The epigenetic regulation of senescent MSCs becomes further tangled, since over 25 miRNAs seem to be influenced by DNAm upon senescence [47]. Interestingly, the number of hypermethylated miRNAs increases during long-term culture, including let-7e, mir-27b, mir-411, or mir-195, triggering downregulation of DNA replication and cell cycle genes. On the other way around, some miRNAs - such as miR-371 and miR-369-5p-are involved in the modulation of the DNA methyl transferases DNMT3A and DNMT3B [68]. Another miRNA involved in the differentiation capacity of MSCs is miR-335, which is upregulated 
during replicative senescence and has been considered a biomarker candidate for the therapeutic potential of MSCs in clinical applications [69]. Furthermore, miR-486-5p shows senescence-associated changes in MSCs and induces replicative senescence of MSCs since it regulates expression of silent information regulator 1 (SIRT1), a major regulator of longevity and metabolic disorders. miR-486-5p expression is controlled by high glucose, connecting age-associated metabolic disorders, miRNA regulation, and senescence of human MSCs [70]. Thus, miRNAs are involved in the induction and preservation of the senescent state mainly by regulating different components of the cell cycle or nuclear organization.

A crucial function in senescence-associated gene regulation is also assigned to another class of ncRNAs: the long non-coding RNAs (lncRNAs). LncRNAs are transcripts that lack protein-coding capacity and range from 200 bases to hundreds of kilobases. However, the role of the lncRNAs in cellular aging is still poorly understood, and it has not yet been addressed in MSCs. In primary human fibroblasts and prostate cancer cell lines, the lncRNA ANRIL (antisense non-coding RNA in the INK4 locus) associates with polycomb repressive complexes (PRCs) PRC1 and PRC2 through chromodomain proteins chromobox homolog 7 (CBX7) and suppressor of zeste 12 protein homolog (SUZ12) to repress the senescence induction of the INK4 locus $[71,72]$. The lncRNA p21associated ncRNA DNA damage activated (PANDA) associates as well to PRC1 and PRC2 for the regulation of senescence [73•]. Also, HOX antisense intergenic RNA (HOTAIR) targets many different loci for PRC2-dependent silencing [74], and this lncRNA is regulated by other epigenetic factors such as the trimeric complex containing AGO2, miR-let7, and RNA-binding protein $\mathrm{Hu}$ antigen $\mathrm{R}$ (HuR). Finally, the IncRNA TERRA, which recruits shelterin to telomeres in a transcription-dependent manner to maintain the telomere length [75], is declined during senescence [27].

These findings indicate that lncRNAs play an important role in regulation of senescence - but it remains to be demonstrated for MSCs.

\section{Conclusions}

Epigenetic mechanisms orchestrate cellular senescence of MSCs. Chromatin organization, histone posttranslational modifications, DNA methylation, and non-coding RNAs act in concert to provide gene expression patterns essential for cell proliferation and cellular function.

However, our current knowledge of the different epigenetic mechanisms involved in senescence of MSCs remains incomplete. While changes in miRNA expression and DNAmethylation are relatively well characterized in senescent MSCs, there is hardly any knowledge about different histone modifications and lncRNAs. Furthermore, the high complexity of interactions between different epigenetic mechanisms still needs to be unraveled. Taken together, the epigenetic modifications provide a fruitful avenue to decipher and understand the senescence regulation in MSCs.

Acknowledgments This work was supported by the Else KrönerFresenius Stiftung (2014_A193), by the German Ministry of Education and Research (BMBF; OBELICS), and the START-Program of the Faculty of Medicine, RWTH Aachen.

\section{Compliance with Ethical Standards}

Conflict of Interest Wolfgang Wagner is cofounder of Cygenia $\mathrm{GmbH}$ (www.cygenia.com). Julia Franzen and Eduardo Fernandez-Rebollo declare that they have no conflict of interest.

Human and Animal Rights and Informed Consent This article does not contain any studies with human or animal subjects performed by any of the authors.

\section{References}

Papers of particular interest, published recently, have been highlighted as:

- Of importance

•• Of major importance

1. Trounson A, McDonald C. Stem cell therapies in clinical trials: progress and challenges. Cell Stem Cell. 2015;17(1):11-22.

2. Wagner W, Bork S, Horn P, et al. Aging and replicative senescence have related effects on human stem and progenitor cells. PLoS One. 2009;4(6), e5846.

3. Hayflick L. The limited in vitro lifetime of human diploid cell strains. Exp Cell Res. 1965;37:614-36.

4. Ostler EL, Wallis CV, Aboalchamat B, et al. Telomerase and the cellular lifespan: implications of the aging process. J Pediatr Endocrinol Metab. 2000;13 Suppl 6:1467-76.

5. Dimauro T, David G. Chromatin modifications: the driving force of senescence and aging? Aging (Albany NY). 2009;1(2):182-90.

6. Courtois-Cox S, Jones SL, Cichowski K. Many roads lead to oncogene-induced senescence. Oncogene. 2008;27(20):2801-9.

7. Wagner W, Fernandez-Rebollo E, Frobel J. DNA-methylation changes in replicative senescence and aging: two sides of the same coin? Epigenomics. 2016;8(1):1-3.

8. Campisi J. Aging, cellular senescence, and cancer. Annu Rev Physiol. 2013;75:685-705.

9. Kulju KS, Lehman JM. Increased p53 protein associated with aging in human diploid fibroblasts. Exp Cell Res. 1995;217(2):336-45.

10. Narita M, Nunez S, Heard E, et al. Rb-mediated heterochromatin formation and silencing of E2F target genes during cellular senescence. Cell. 2003;113(6):703-16.

11.• Lopez-Otin C, Blasco MA, Partridge L, et al. The hallmarks of aging. Cell. 2013;153(6):1194-217. This review summarizes the common hallmarks of aging in different organisms.

12. Bickmore WA, van Steensel B. Genome architecture: domain organization of interphase chromosomes. Cell. 2013;152(6):1270-84.

13. Collas P, Lund EG, Oldenburg AR. Closing the (nuclear) envelope on the genome: how nuclear lamins interact with promoters and modulate gene expression. Bioessays. 2014;36(1):75-83. 
14. Chandra T, Ewels PA, Schoenfelder S, et al. Global reorganization of the nuclear landscape in senescent cells. Cell Rep. 2015;10(4): 471-83.

15. Criscione SW, De Cecco M, Siranosian B, et al. Reorganization of chromosome architecture in replicative cellular senescence. Sci Adv. 2016;2(2), e1500882.

16. Raz V, Vermolen BJ, Garini Y, et al. The nuclear lamina promotes telomere aggregation and centromere peripheral localization during senescence of human mesenchymal stem cells. J Cell Sci. 2008;121(Pt 24):4018-28.

17. Righolt $\mathrm{CH}$, van't Hoff ML, Vermolen BJ, et al. Robust nuclear lamina-based cell classification of aging and senescent cells. Aging (Albany NY). 2011;3(12):1192-201.

18. Hanzelmann S, Beier F, Gusmao EG, et al. Replicative senescence is associated with nuclear reorganization and with DNA methylation at specific transcription factor binding sites. Clin Epigenetics. 2015;7(1):19. In this research article, the authors analyze the reorganization of nuclear architecture and DNAm changes during long-term culture of human fibroblasts and mesenchymal stromal cells.

19. Cruickshanks HA, Mcbryan T, Nelson DM, et al. Senescent cells harbour features of the cancer epigenome. Nat Cell Biol. 2013;15(12):1495-506.

20. Zhang W, Li J, Suzuki K, et al. A Werner syndrome stem cell model unveils heterochromatin alterations as a driver of human aging. Science. 2015;348(6239):1160-3. This research article describes that the genetic mutations underlying Werner syndrome, a disorder that leads to premature aging, result in the loss of nuclear organization.

21. Alessio N, Squillaro T, Cipollaro M, et al. The BRG1 ATPase of chromatin remodeling complexes is involved in modulation of mesenchymal stem cell senescence through RB-P53 pathways. Oncogene. 2010;29(40):5452-63.

22. Napolitano MA, Cipollaro M, Cascino A, et al. Brg1 chromatin remodeling factor is involved in cell growth arrest, apoptosis and senescence of rat mesenchymal stem cells. J Cell Sci. 2007;120(16):2904-11.

23. Squillaro T, Severino V, Alessio N, et al. De-regulated expression of the BRG1 chromatin remodeling factor in bone marrow mesenchymal stromal cells induces senescence associated with the silencing of NANOG and changes in the levels of chromatin proteins. Cell Cycle. 2015;14(8):1315-26.

24. Blackburn EH. Switching and signaling at the telomere. Cell. 2001;106(6):661-73.

25. de Lange T. Shelterin: the protein complex that shapes and safeguards human telomeres. Genes Dev. 2005;19(18):2100-10.

26. Martinez P, Blasco MA. Telomeric and extra-telomeric roles for telomerase and the telomere-binding proteins. Nat Rev Cancer. 2011;11(3):161-76.

27. Caslini C, Connelly JA, Serna A, et al. MLL associates with telomeres and regulates telomeric repeat-containing RNA transcription. Mol Cell Biol. 2009;29(16):4519-26.

28. Simonsen JL, Rosada C, Serakinci N, et al. Telomerase expression extends the proliferative life-span and maintains the osteogenic potential of human bone marrow stromal cells. Nat Biotechnol. 2002;20(6):592-6.

29. Bocker W, Yin Z, Drosse I, et al. Introducing a single-cell-derived human mesenchymal stem cell line expressing hTERT after lentiviral gene transfer. J Cell Mol Med. 2008;12(4):1347-59.

30. Zhao Q, Wang XY, Yu XX, et al. Expression of human telomerase reverse transcriptase mediates the senescence of mesenchymal stem cells through the PI3K/AKT signaling pathway. Int J Mol Med. 2015;36(3):857-64.

31. Ben-David U, Mayshar Y, Benvenisty N. Large-scale analysis reveals acquisition of lineage-specific chromosomal aberrations in human adult stem cells. Cell Stem Cell. 2011;9(2):97-102.
32. Laurent LC, Ulitsky I, Slavin I, et al. Dynamic changes in the copy number of pluripotency and cell proliferation genes in human ESCs and iPSCs during reprogramming and time in culture. Cell Stem Cell. 2011;8(1):106-18.

33. Estrada JC, Torres Y, Benguria A, et al. Human mesenchymal stem cell-replicative senescence and oxidative stress are closely linked to aneuploidy. Cell Death Dis. 2013;4:e691. In this research article, the authors address the relevance of the aneuploidy as a genomic inastibility mechanism triggered upon replicative senescence and oxidative stress in human mesenchymal stem cells.

34. Wagner JR, Busche S, Ge B, et al. The relationship between DNA methylation, genetic and expression inter-individual variation in untransformed human fibroblasts. Genome Biol. 2014;15(2):R37.

35. Koch CM, Suschek CV, Lin Q, et al. Specific age-associated DNA methylation changes in human dermal fibroblasts. PLoS One. 2011;6(2), e16679.

36. de Almeida DC, Ferreira MRP, Franzen J, et al. Epigenetic classification of human mesenchymal stromal cells. Stem Cell Reports. 6(2): p. 168-175.

37. Koch CM, Reck K, Shao K, et al. Pluripotent stem cells escape from senescence-associated DNA methylation changes. Genome Res. 2013;23(2):248-59. In this research article, the authors study specific senescence-associated DNA methylation (SA-DNAm) changes in mesenchymal stem cells upon different insults triggering senescence.

38. Bork S, Pfister S, Witt H, et al. DNA methylation pattern changes upon long-term culture and aging of human mesenchymal stromal cells. Aging Cell. 2010;9(1):54-63.

39. Schellenberg A, Mauen S, Koch CM, et al. Proof of principle: quality control of therapeutic cell preparations using senescenceassociated DNA-methylation changes. BMC Res Notes. 2014;7: 254.

40. Koch CM, Joussen S, Schellenberg A, et al. Monitoring of cellular senescence by DNA-methylation at specific $\mathrm{CpG}$ sites. Aging Cell. 2012;11(2):366-9.

41. Weidner CI, Lin Q, Koch CM, et al. Aging of blood can be tracked by DNA methylation changes at just three $\mathrm{CpG}$ sites. Genome Biol. 2014;15(2):R24.

42. Hannum G, Guinney J, Zhao L, et al. Genome-wide methylation profiles reveal quantitative views of human aging rates. Mol Cell. 2013;49(2):359-67.

43. Horvath S. DNA methylation age of human tissues and cell types. Genome Biol. 2013;14(10):R115.

44. Frobel J, Hemeda H, Lenz M, et al. Epigenetic rejuvenation of mesenchymal stromal cells derived from induced pluripotent stem cells. Stem Cell Rep. 2014;3(3):414-22. In this research article, the authors analyze the DNAm patterns in iPSCs reprogrammed from MSCs and redifferentiated iPSC derived MSCs.

45. Despars G, Carbonneau CL, Bardeau P, et al. Loss of the osteogenic differentiation potential during senescence is limited to bone progenitor cells and is dependent on p53. PLoS One. 2013;8(8), e73206.

46. Noer A, Boquest AC, Collas P. Dynamics of adipogenic promoter DNA methylation during clonal culture of human adipose stem cells to senescence. BMC Cell Biology. 2007;8:18-8.

47. Choi MR, In YH, Park J, et al. Genome-scale DNA methylation pattern profiling of human bone marrow mesenchymal stem cells in long-term culture. Exp Mol Med. 2012;44(8):503-12.

48. Li Z, Liu C, Xie Z, et al. Epigenetic dysregulation in mesenchymal stem cell aging and spontaneous differentiation. PLoS One. 2011;6(6), e20526.

49. Anderson DC, Green GR, Smith K, et al. Extensive and varied modifications in histone $\mathrm{H} 2 \mathrm{~B}$ of wild-type and histone deacetylase 1 mutant Neurospora crassa. Biochemistry. 2010;49(25):5244-57. 
50. Cosgrove MS, Wolberger C. How does the histone code work? Biochem Cell Biol. 2005;83(4):468-76.

51. Bhaumik SR, Smith E, Shilatifard A. Covalent modifications of histones during development and disease pathogenesis. Nat Struct Mol Biol. 2007;14(11):1008-16.

52. Lee S, Park JR, Seo MS, et al. Histone deacetylase inhibitors decrease proliferation potential and multilineage differentiation capability of human mesenchymal stem cells. Cell Prolif. 2009;42(6): 711-20.

53. Agalioti T, Chen G, Thanos D. Deciphering the transcriptional histone acetylation code for a human gene. Cell. 2002;111(3):381-92.

54. Lomvardas $\mathrm{S}$, Thanos D. Nucleosome sliding via TBP DNA binding in vivo. Cell. 2001;106(6):685-96.

55. Jung JW, Lee S, Seo MS, et al. Histone deacetylase controls adult stem cell aging by balancing the expression of polycomb genes and jumonji domain containing 3. Cell Mol Life Sci. 2010;67(7):116576.

56. Noer A, Lindeman LC, Collas P. Histone H3 modifications associated with differentiation and long-term culture of mesenchymal adipose stem cells. Stem Cells Dev. 2009;18(5):725-36.

57. Schellenberg A, Lin Q, Schuler H, et al. Replicative senescence of mesenchymal stem cells causes DNA-methylation changes which correlate with repressive histone marks. Aging (Albany NY). 2011;3(9):873-88.

58. Fernandez AF, Bayon GF, Urdinguio RG, et al. H3K4me1 marks DNA regions hypomethylated during aging in human stem and differentiated cells. Genome Res. 2015;25(1):27-40. In this research article, the authors profile the DNA methylation changes and histone marks of mesenchymal stem cells from young and old donors, finding that hypomethylated $\mathrm{CpG}$ sites were strongly enriched in the active chromatin mark H3K4me1.

59. Chen K, Rajewsky N. The evolution of gene regulation by transcription factors and microRNAs. Nat Rev Genet. 2007;8(2):93103.

60. Lewis BP, Shih IH, Jones-Rhoades MW, et al. Prediction of mammalian microRNA targets. Cell. 2003;115(7):787-98.

61. Wagner W, Horn P, Castoldi M, et al. Replicative senescence of mesenchymal stem cells: a continuous and organized process. PLoS One. 2008;3(5), e2213.

62. Zou Z, Zhang Y, Hao L, et al. More insight into mesenchymal stem cells and their effects inside the body. Expert Opin Biol Ther. 2010;10(2):215-30.

63. Yoo JK, Kim J, Choi SJ, et al. Discovery and characterization of novel microRNAs during endothelial differentiation of human embryonic stem cells. Stem Cells Dev. 2012;21(11):2049-57.
64. Benhamed M, Herbig U, Ye T, et al. Senescence is an endogenous trigger for microRNA-directed transcriptional gene silencing in human cells. Nat Cell Biol. 2012;14(3):266-75.

65. Li J, Dong J, Zhang ZH, et al. miR-10a restores human mesenchymal stem cell differentiation by repressing KLF4. J Cell Physiol. 2013;228(12):2324-36.

66. Yoo JK, Kim CH, Jung HY, et al. Discovery and characterization of miRNA during cellular senescence in bone marrow-derived human mesenchymal stem cells. Exp Gerontol. 2014;58:139-45. In this research article, the authors identify and characterize several miRNAs during cellular senescence in mesenchymal stem cells.

67. Peffers MJ, Collins J, Fang Y, et al. Age-related changes in mesenchymal stem cells identified using a multi-omics approach. Eur Cell Mater. 2016;31:136-59.

68. Bork S, Horn P, Castoldi M, et al. Adipogenic differentiation of human mesenchymal stromal cells is down-regulated by microRNA-369-5p and up-regulated by microRNA-371. J Cell Physiol. 2011;226(9):2226-34.

69. Tome M, Sepulveda JC, Delgado M, et al. miR-335 correlates with senescence/aging in human mesenchymal stem cells and inhibits their therapeutic actions through inhibition of AP-1 activity. Stem Cells. 2014;32(8):2229-44.

70. Kim YJ, Hwang SH, Lee SY, et al. miR-486-5p induces replicative senescence of human adipose tissue-derived mesenchymal stem cells and its expression is controlled by high glucose. Stem Cells Dev. 2012;21(10):1749-60.

71. Yap KL, Li S, Munoz-Cabello AM, et al. Molecular interplay of the noncoding RNA ANRIL and methylated histone H3 lysine 27 by polycomb CBX7 in transcriptional silencing of INK4a. Mol Cell. 2010;38(5):662-74.

72. Kotake Y, Nakagawa T, Kitagawa K, et al. Long non-coding RNA ANRIL is required for the PRC2 recruitment to and silencing of p15(INK4B) tumor suppressor gene. Oncogene. 2011;30(16): 1956-62.

73. Puvvula PK, Desetty RD, Pineau P, et al. Long noncoding RNA PANDA and scaffold-attachment-factor SAFA control senescence entry and exit. Nat Commun. 2014;5:5323. In this research article, the authors describe the role of the long noncoding RNA PANDA upon senescence.

74. Rinn JL, Kertesz M, Wang JK, et al. Functional demarcation of active and silent chromatin domains in human HOX loci by noncoding RNAs. Cell. 2007;129(7):1311-23.

75. Caslini C. Transcriptional regulation of telomeric non-coding RNA: implications on telomere biology, replicative senescence and cancer. RNA Biol. 2010;7(1):18-22. 